\title{
Evaluating the prevalence of diabetes mellitus subtypes in childhood cancer survivors: a systematic review protocol
}

This article was published in the following Dove Press journal:

Adolescent Health, Medicine and Therapeutics

\author{
Sondra Song Jie Chen ${ }^{1,2}$ \\ Ajantha Nadarajah ${ }^{1,2}$ \\ Laura Banfield ${ }^{3}$ \\ Adam Fleming ${ }^{1,4}$ \\ Lehana Thabane ${ }^{5-8}$ \\ Carol Portwine ${ }^{1,4}$ \\ M Constantine Samaan 1,2,5 \\ 'Department of Pediatrics, McMaster \\ University, Hamilton, Ontario, Canada; \\ ${ }^{2}$ Division of Pediatric Endocrinology, \\ McMaster Children's Hospital, Hamilton, \\ Ontario, Canada; ${ }^{3}$ Health Sciences \\ Library, McMaster University, Hamilton, \\ Ontario, Canada; ${ }^{4}$ Division of Pediatric \\ Hematology/Oncology, McMaster \\ Children's Hospital, Hamilton, Ontario, \\ Canada; ${ }^{5}$ Department of Health Research \\ Methods, Evidence and Impact, McMaster \\ University, Hamilton, Ontario, Canada; \\ ${ }^{6}$ Department of Anesthesia, McMaster \\ University, Hamilton, Ontario, Canada; \\ ${ }^{7}$ Centre for Evaluation of Medicines, St \\ Joseph's Health Care, Hamilton, Ontario, \\ Canada; ${ }^{8}$ Biostatistics Unit, St Joseph's \\ Healthcare, Hamilton, Ontario, Canada
}

Correspondence: M Constantine Samaan 1280 Main Street West, HSC-3A, Hamilton, Ontario L8S 4KI, Canada Tel + I 9055212100 Ext. 75926

$\mathrm{Fax}+19053087548$

Email samaanc@mcmaster.ca

\begin{abstract}
Objectives: The number of children who survive cancer is reaching new record levels, thanks to improved management strategies. However, this population is predisposed to chronic health conditions including cardiovascular disease and type 2 diabetes, yet the full scale of these diagnoses in this population is unclear. This protocol describes the conduct of a systematic review to report on the prevalence of diabetes mellitus (DM) subtypes in childhood cancer survivors.
\end{abstract}

Methods: Searches will be conducted in MEDLINE, Embase, CINAHL, Cochrane Database of Systematic Reviews, and Cochrane Central Register of Controlled Trials. We will also search gray literature in Theses A\&I, ProQuest Dissertations, and Web of Science as well as clinicaltrials.gov. Screening search results and data abstraction will be done independently by two reviewers. We will conduct a meta-analysis if two studies have similar designs, populations, methods, and outcome measures reported.

Results: The findings of this systematic review will provide insights into the scale of diabetes in childhood cancer survivors to allow the prioritization of subpopulations that need specific interventions to screen, prevent, and treat DM. This will likely lead to improved outcomes in childhood cancer survivors.

Keywords: systematic review protocol, childhood cancer, cancer survivorship, diabetes mellitus, type 1 diabetes mellitus, type 2 diabetes mellitus

\section{Introduction}

Over the past few decades, breakthroughs in childhood cancer care have considerably improved the five-year survival rates in this population. In $1979,62.8 \%$ of children survived their cancer diagnosis, and this rose to $84.4 \%$ in $2014 .{ }^{1}$ These impressive survival rates, however, are mitigated by the development of significant comorbidities that interfere with long-term outcomes. The majority of Childhood Cancer Survivors (CCS) have at least one chronic health condition in adulthood. A new class of emerging morbidities including diabetes mellitus (DM), cardiovascular diseases, and obesity are being added to causes of morbidity and mortality in CCS such as tumor recurrence and secondary cancers. ${ }^{2-11}$ Cardiometabolic disorders are taking a more prominent role in determining long-term outcomes in addition to lifestyle factors, tumor type, and treatment side-effects, among others. $^{10,12-15}$ Several pathways can lead to diabetes in CCS including defects in insulin production, insulin insensitivity in peripheral organs, and other factors that remain to be defined. 
The global rates of diabetes have reached staggering levels, with around 422 million people worldwide having type 2 diabetes mellitus (T2DM) in 2014, and the obesity epidemic has driven this rise. ${ }^{16}$ It has been shown that CCS have similar rates of obesity but higher adiposity when compared to the general population, which may impact their diabetes risk through effects of adiposity on glucose homeostasis. ${ }^{17}$ Diabetes represents a substantial economic and societal burden and is associated with several comorbidities including retinopathy, nephropathy, neuropathy and cardiovascular disease. ${ }^{18}$ It is critical to determine the burden of DM and if, within the CCS cohort, there are particularly vulnerable subgroups that may benefit from offering early screening and interventions to treat, delay, and prevent DM in these populations.

This protocol describes the methods used for a systematic review to assess the prevalence of DM in CCS. The primary objective is to determine the prevalence of T2DM in CCS. The secondary objectives are to report the prevalence of type 1 diabetes mellitus (T1DM) and undifferentiated DM (UDM) in CCS.

\section{Research questions}

Primary

In CCS, what is the prevalence of T2DM?

\section{Secondary}

In CCS, what is the prevalence of T1DM and UDM?

\section{Materials and methods}

This systematic review protocol is registered with PROSPERO (CRD42018090456).

This protocol is reported based on the Preferred Reporting Items for Systematic Review and Meta-Analysis Protocols (PRISMA-P) Statement (Supplementary materials). ${ }^{19,20}$

\section{Eligibility criteria Population}

We will include males and females of all ethnicities who have received a cancer diagnosis at $\leq 20$ years of age. All Body Mass Index (BMI) levels will be included, comprising those with normal BMI (BMI z-score $<85$ th percentile), overweight (BMI z-score $85^{\text {th }}-94^{\text {th }}$ percentile), and obese (BMI z-score $\geq 95$ th percentile).

We will exclude studies with a sample size of less than 10 participants. If a study reports aggregated data from survivors of both childhood and adult cancers, the
Principal Investigator (PI) of the study will be contacted to obtain the age-stratified data specific to our inclusion criteria. Diabetes is required to have developed at least one year after the completion of cancer therapy for studies to be included in this systematic review.

\section{Exposure}

Survivors of all cancer types will be included, with cancer defined as per the International Classification of Childhood Cancer. $^{21}$ This diagnosis may be obtained from secure medical records, patient interviews, or questionnaires. We will collect data on therapy including surgery, pharmacotherapy, radiotherapy, and transplantation. ${ }^{22}$ Surgery types will include total or partial tumor resection. All forms of chemotherapy will be included, as well as data on the use of steroids and immunotherapies. For radiation-sensitive tumors, we will include all forms of radiotherapy including total body irradiation, craniospinal irradiation, or cranial irradiation with non-fractionated and fractionated subgroups. Studies reporting on patients with gestational DM will be excluded. $^{11}$

\section{Comparator}

If reported, we will include non-cancer controls from the general population that are matched or unmatched to cases. Alternatively, family-based controls (eg siblings) as a matching method for controls will be included if reported. $^{23}$

\section{Outcome}

We will include all types of DM including T1DM, T2DM, and UDM.

The DM diagnosis should be confirmed by one of the following methods:

1. Hospital records

2. The diagnosis determined from patient interviews

3. The diagnosis documented by health registries or other databases via record linkage

4. Confirmation of healthcare provider reporting or billing for a DM diagnosis

5. Results from fasting or random blood glucose, oral glucose tolerance tests, and $\mathrm{HbAlc}$ along with T1DM antibodies

6. Patient surveys confirming the implementation of lifestyle-based approaches to treat mainly T2DM

7. Patient or provider reporting of oral antihyperglycemic therapies or insulin usage 


\section{Study designs}

Observational studies (cohort, cross-sectional or casecontrol studies) will be included in this review. There will be no restrictions on the date of publication, geographical location, study setting, or language of publication.

\section{Literature search}

A Senior Health Sciences Librarian with proficiency in methods used in systematic review search strategy development and implementation will assist in developing the search strategy. The searches will be conducted in MEDLINE, Experta Medica dataBASE (Embase), the Cumulative Index to Nursing and Allied Health Literature (CINAHL), Cochrane Database of Systematic Reviews, and Cochrane Central Register of Controlled Trials. A sample search strategy for MEDLINE is provided in Table 1.

We will hand search reference lists of accepted studies and relevant reviews for additional eligible studies. To identify gray literature ProQuest Dissertations, Theses A\&I, clinicaltrials.gov and Web of Science will be searched. While conference abstracts will be excluded, we will search the first and last authors of any relevant abstract for publications that originate from the work provided. Searches will be run up to and including studies published in March 2019.

\section{Study selection}

We will report the screening procedure and results using the Preferred Reporting Items for Systematic Reviews and Meta-Analyses (PRISMA) guidelines. ${ }^{20,24,25}$ A preliminary version of the flow diagram for the reporting of this review is presented in Figure 1.

Duplicate references will be removed using EndNote X8 and refernces exported into Microsoft Excel to screen titles, abstracts, and full-text publications by two independent reviewers. ${ }^{26}$ The reviewers will meet after each stage to assess agreement and will resolve disagreements through discussion. If the conflict persists, a third independent reviewer will be consulted to reach unanimity.

\section{Data abstraction}

A data abstraction form will be created and piloted by two reviewers on a sample subset of publications for this review. Data to be collected include study title, authors, journal name, publication date, country, funding source, study setting, design, duration, years of conduct, inclusion and exclusion criteria, sample size, outcome measures, primary findings, secondary outcomes, conclusions, and
Table I Search strategy for MEDLINE

\begin{tabular}{|c|c|}
\hline Line & Search terms \\
\hline $\mathrm{I}$ & $\exp$ Child/ \\
\hline 2 & exp Adolescent/ \\
\hline 3 & child*.ab,ti,kf. \\
\hline 4 & p?ediatric*.ab,ti,kf. \\
\hline 5 & adolescen*.ab,ti,kf. \\
\hline 6 & youth*.ab,ti,kf. \\
\hline 7 & teen*.ab,ti,kf. \\
\hline 8 & preteen*.ab,ti,kf. \\
\hline 9 & tween*.ab,ti,kf. \\
\hline 10 & preschool*.ti,ab,kf. \\
\hline $\mathrm{II}$ & kindergarten*.ti,ab,kf. \\
\hline 12 & school-aged.ti,ab,kf. \\
\hline 13 & (school adj3 age*).ti,ab,kf. \\
\hline 14 & (young adj3 adult).ti,ab,kf. \\
\hline 15 & or/I-14 \\
\hline 16 & exp Diabetes Mellitus/ \\
\hline 17 & exp Hyperglycemia/ \\
\hline 18 & glycosuria/ \\
\hline 19 & NIDDM.ti,ab,kf. \\
\hline 20 & IDDM.ti,ab,kf. \\
\hline 21 & T2D*.ti,ab,kf. \\
\hline 22 & TID*.ti,ab,kf. \\
\hline 23 & hyperglycem*.ti,ab,kf. \\
\hline 24 & diabet*.ti,ab,kf. \\
\hline 25 & (insulin* adj3 resist*).ti,ab,kf. \\
\hline 26 & $\begin{array}{l}\text { ((non-insulin* or noninsulin* or non insulin*) adj3 resist*).ti, } \\
\text { ab,kf. }\end{array}$ \\
\hline 27 & (insulin* adj3 depend*).ti,ab,kf. \\
\hline 28 & $\begin{array}{l}\text { ((non-insulin* or noninsulin* or non insulin*) adj3 depend*). } \\
\text { ti,ab,kf. }\end{array}$ \\
\hline 29 & hyperinsulin*.ti,ab,kf. \\
\hline 30 & hypoinsulin*.ti,ab,kf. \\
\hline 31 & hypertriglyceridem*.ti,ab,kf. \\
\hline 32 & (dyslipid* or hyperlipid*).ti,ab,kf. \\
\hline 33 & ketoacid*.ti,ab,kf. \\
\hline 34 & (glucose adj3 (toleran* or intoleran*)).ti,ab,kf. \\
\hline 35 & or/16-34 \\
\hline 36 & 15 and 35 \\
\hline 37 & Survivors/ \\
\hline 38 & Survival/ \\
\hline 39 & Adult Survivors of Child Adverse Events/ \\
\hline 40 & Disease-Free Survival/ \\
\hline 41 & surviv*.ab,ti,kf. \\
\hline 42 & ((post or off or after) adj3 (cancer* or tumo?r*)).ab,ti,kf. \\
\hline 43 & ((post or off or after) adj3 (treatment* or therap*)).ab,ti,kf. \\
\hline 44 & $\begin{array}{l}((\text { treatment* or therap* or cancer* or disease* or event* or } \\
\text { progression*) adj4 free).ab,ti,kf. }\end{array}$ \\
\hline 45 & (adult life adj5 child* cancer).ti,ab,kf. \\
\hline 46 & remission*.ti,ab,kf. \\
\hline 47 & (cancer free* or cancer-free*).ti,ab,kf. \\
\hline 48 & (after adj2 cancer*).ti,ab,kf. \\
\hline
\end{tabular}

(Continued) 
Table I (Continued).

\begin{tabular}{|c|c|}
\hline Line & Search terms \\
\hline 49 & (after adj3 tumo? r*).ti,ab,kf. \\
\hline 50 & (late effect* or late-effect*).ti,ab,kf. \\
\hline 51 & or/37-50 \\
\hline 52 & 36 and $5 I$ \\
\hline 53 & exp Neoplasms/ \\
\hline 54 & cancer*.ti,ab,kf. \\
\hline 55 & tumo?r.ti,ab,kf. \\
\hline 56 & malignan*.ti,ab,kf. \\
\hline 57 & exp Leukemia/ \\
\hline 58 & exp Lymphoma, Non-Hodgkin/or exp Lymphoma/ \\
\hline 59 & exp Brain Neoplasms/ \\
\hline 60 & exp Sarcoma/ \\
\hline 61 & exp Kidney Neoplasms/ \\
\hline 62 & $\begin{array}{l}\text { exp Adenoma, Islet Cell/or exp Neuroendocrine Tumors/or } \\
\text { exp Pancreatic Neoplasms/or exp Thyroid Neoplasms/ }\end{array}$ \\
\hline 63 & exp Bone Neoplasms/ \\
\hline 64 & exp Testicular Neoplasms/or exp Ovarian Neoplasms/ \\
\hline 65 & retinoblastoma.ti,ab,kf. \\
\hline 66 & leuk?emia*.ti,ab,kf. \\
\hline 67 & leucocyth?emia*.ti,ab,kf. \\
\hline 68 & myelo*.ti,ab,kf. \\
\hline 69 & lymphoblas*.ti,ab,kf. \\
\hline 70 & lymphoma*.ti,ab,kf. \\
\hline 71 & hodgkin*.ti,ab,kf. \\
\hline 72 & non-hodgkin*.ti,ab,kf. \\
\hline 73 & germinoblastoma*.ti,ab,kf. \\
\hline 74 & reticulolymphosarcoma*.ti,ab,kf. \\
\hline 75 & reticulosarcoma*.ti,ab,kf. \\
\hline 76 & $\exp$ Neuroectodermal Tumors, Primitive/ \\
\hline 77 & exp Glioma/ \\
\hline 78 & glioma*.ti,ab,kf. \\
\hline 79 & astrocytoma*.ab,ti,kf. \\
\hline 80 & oligoastrocytoma*.ti,ab,kf. \\
\hline 81 & astroglioma*.ti,ab,kf. \\
\hline 82 & glioblastoma*.ti,ab,kf. \\
\hline 83 & craniopharyngioma*.ti,ab,kf. \\
\hline 84 & ependymoma*.ab,ti,kf. \\
\hline 85 & subependymoma*.ab,ti,kf. \\
\hline 86 & ependymoblastoma*.ab,ti,kf. \\
\hline 87 & ganglioglioma*.ab,ti,kf. \\
\hline 88 & medulloblastoma*.ab,ti,kf. \\
\hline 89 & $\exp$ Germinoma/ \\
\hline 90 & germinoma*.ab,ti,kf. \\
\hline 91 & Meningioma/ \\
\hline 92 & meningioma*.ab,ti,kf. \\
\hline 93 & oligodendroglioma*.ab,ti,kf. \\
\hline 94 & exp Neurofibromatoses/ \\
\hline 95 & neurofibromatos*.ab,ti,kf. \\
\hline 96 & PNET*.ab,ti,kf. \\
\hline 97 & neurocytoma*.ab,ti,kf. \\
\hline 98 & choroid plexus papilloma*.ab,ti,kf. \\
\hline
\end{tabular}

(Continued)
Table I (Continued).

\begin{tabular}{|l|l|}
\hline Line & Search terms \\
\hline 99 & sarcoma*.ti,ab,kf. \\
I00 & neuroblastoma*.ti,ab,kf. \\
101 & exp Wilms Tumor/ \\
102 & nephroblastoma*.ti,ab,kf. \\
103 & (wilms* adj3 tumo?r).ti,ab,kf. \\
104 & Rhabdomyosarcoma*.ti,ab,kf. \\
105 & osteosarcoma*ti,ab,kf. \\
106 & chondrosarcoma*.ti,ab,kf. \\
107 & neoplas*.ti,ab,kf. \\
108 & exp Carcinoma/ \\
109 & carcinoma*.ti,ab,kf. \\
II0 & adenocarcinoma*.ti,ab,kf. \\
III & epithelioma*.ti,ab,kf. \\
II2 & or/53-III \\
II3 & 52 and II2 \\
II4 & Animals/not (humans/and animals/) \\
II5 & II3 not II4 \\
II6 & Remove duplicates from II5 \\
\hline
\end{tabular}

relevant study-specific details. We will also extract survivors' data including the age at cancer diagnosis, the age of study enrolment, sex, ethnicity, and associated comorbidities. Details of cancer type, stage at diagnosis, and treatment modalities and duration since treatment completion will also be extracted. If there is a comparison group, reviewers will extract the type of comparison group followed by the same clinical and health-related data (except cancer diagnosis and treatment data) as the CCS group. If aggregate data are presented, we will reach out to the study PI to retrieve data specific to our population.

For DM diagnoses, reviewers will extract information about the patients' age at diagnosis, the time between cancer treatment completion and DM diagnosis, DM type, and study-specific prevalence estimates of DM subtypes in the survivorship population. We will abstract similar data from the non-cancer group if reported.

\section{Data synthesis and statistical analysis}

A meta-analysis will be performed if two or more studies reporting an outcome have similar study design, populations, methods, and outcomes. This analysis will be conducted to generate an estimate of the prevalance of diabetes subtypes from eligible studies by reporting the point prevalence from the pooled studies among CCS and any CCS subgroups (eg age at cancer diagnosis, sex, cancer type, cancer treatment, 


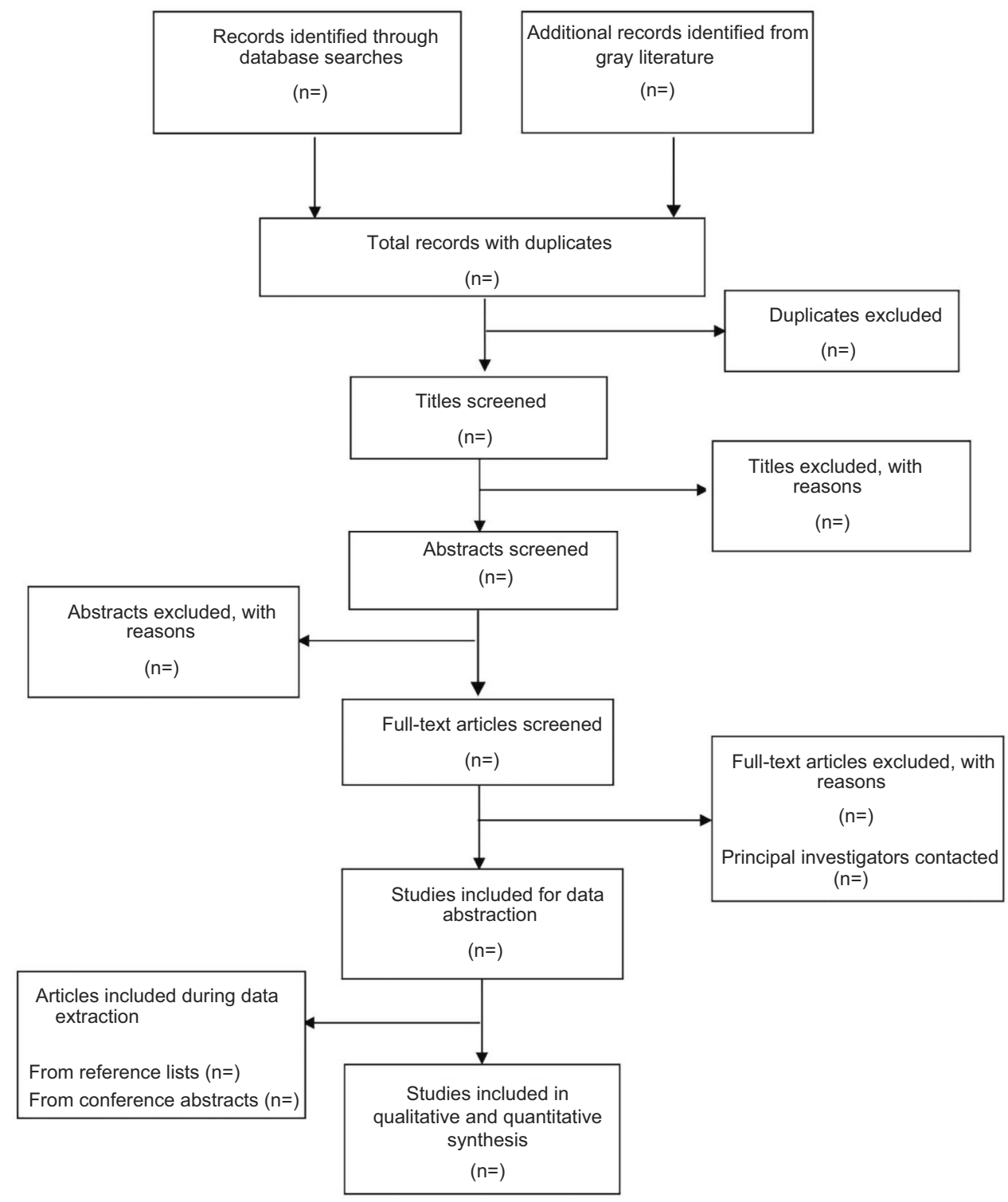

Figure I PRISMA-flow diagram of the article screening process.

body mass index (BMI)). High heterogeneity is expected due to the likely variation among populations and the presence of comorbidities such as obesity. We will use a random effects model for all outcomes. Risk of bias will be conducted using the tool developed by Hoy et al. ${ }^{27}$

Our overall outcome of prevalence is continuous and will be reported as pooled prevalence with a $95 \%$ confidence interval (CI). If studies report multiple measurements for groups over time, we will include the outcomes with the most extended follow-up period. We will also compare the prevalence between CCS and controls by calculating odds ratios. Pooled prevalence estimates from meta-analyses will be summarized and presented using forest plots if applicable.
The inconsistency index $\left(\mathrm{I}^{2}\right)$ and p-value for the chisquare test will be used to assess heterogeneity. An $\mathrm{I}^{2}$ value $>75 \%$ and $p$-value of $<0.1$ will be the threshold for considerable heterogeneity, respectively. ${ }^{28}$ If a metaanalysis cannot be performed, we will provide a comprehensive narrative summary describing the studies. Subgroup analyses will be performed for age at cancer diagnosis, sex, cancer type, cancer treatment, and BMI if reported. Sensitivity analyses will be performed if over ten studies can be identified for a given outcome, to maintain the power of the results. Publication bias will be determined using funnel plots if ten or more studies are identified for the metaanalysis. $^{29}$ 
All analyses will be performed using the Review Manager software (RevMan) Version 5.3. ${ }^{30}$

Overall findings from eligible studies will be reported in a comprehensive table and through a narrative summary, regardless if a meta-analysis is conducted or not.

All results will be documented according to PRISMA Guidelines and reported using the PRISMA checklist. ${ }^{24,25}$ Any amendments to this protocol will be documented with their corresponding rationale in the full review.

\section{Discussion and conclusion}

CCS is an emerging group with chronic health conditions that contribute to future adverse outcomes. ${ }^{1,3,10,11,31,32}$ This systematic review aims to provide insight into the prevalence of DM subtypes in CCS.

There are two potential limitations to the review. The first is a prediction of high heterogeneity among studies, which may impact the results of the meta-analysis if it is applicable to perform. Second, the specific eligibility requirements of reporting DM prevalence may result in fewer eligible studies than predicted. However, the results of this review will provide insights for the scale of diabetes in the CCS population, which will allow the implementation of screening, prevention, and intervention strategies to improve medical treatment and potentially delay or prevent diabetes mellitus, which will likely improve health outcomes in this population.

\section{Abbreviations}

BMI, body mass index; CCS, Childhood Cancer Survivors; CINAHL, Cumulative Index to Nursing and Allied Health Literature; DM, diabetes mellitus; Embase, Excerpta Medica dataBASE; MEDLINE, medical literature analysis and retrieval system online; PI, Principal Investigator; PRISMA, Preferred Reporting Items for Systematic Reviews and Meta-analyses; PRISMA-P, preferred Reporting Items for Systematic Review and Meta-Analysis Protocols; RevMan 5.3, Review Manager version 5.3 software; T1DM, Type 1 diabetes mellitus; T2DM, Type 2 diabetes mellitus; UDM, undifferentiated diabetes mellitus.

\section{Author contributions}

All authors contributed to data analysis, drafting or revising the article, gave final approval of the version to be published, and agree to be accountable for all aspects of the work.

\section{Disclosure}

The authors declare that they have no competing interests in this work.

\section{References}

1. SEER cancer statistics review, 1975-2015. National Cancer Institute; 2017. Accessed January 2018. https://seer.cancer.gov/csr/1975_2015/.

2. Armstrong GT, Oeffinger KC, Chen Y, et al. Modifiable risk factors and major cardiac events among adult survivors of childhood cancer. $J$ Clin Oncol. 2013;31(29):3673-3680. doi:10.1200/JCO.2013.49.3205

3. Oeffinger KC, Mertens AC, Sklar CA, et al. Chronic health conditions in adult survivors of childhood cancer. $N$ Engl J Med. 2006;355 (15):1572-1582. doi:10.1056/NEJMsa060185

4. Van Leeuwen FE, Klokman WJ, Veer MB, et al. Long-term risk of second malignancy in survivors of Hodgkin's disease treated during adolescence or young adulthood. J Clin Oncol. 2000;18 (3):487-497. doi:10.1200/JCO.2000.18.3.487

5. Steinberger J, Sinaiko AR, Kelly AS, et al. Cardiovascular risk and insulin resistance in childhood cancer survivors. J Pediatr. 2012;160 (3):494-499. doi:10.1016/j.jpeds.2011.08.018

6. Chemaitilly W, Sklar CA. Endocrine complications in long-term survivors of childhood cancers. Endocr Relat Cancer. 2010;17(3): R141-159. doi:10.1677/ERC-10-0002

7. Gurney JG, Kadan-Lottick NS, Packer RJ, et al. Endocrine and cardiovascular late effects among adult survivors of childhood brain tumors: childhood cancer survivor study. Cancer. 2003;97 (3):663-673. doi:10.1002/cncr.11095

8. Chao C, Xu L, Bhatia S, Cooper R, BrarS, WongFL, Armenian SH. Cardiovascular disease risk profiles in survivors of adolescent and young adult (AYA) cancer: the Kaiser Permanente AYA Cancer Survivors Study. J Clin Oncol. 2016;34(14):1626-1633. doi:10.1200/JCO.2015.65.5845

9. Heikens J, Ubbink MC, van der Pal HP, et al. Long term survivors of childhood brain cancer have an increased risk for cardiovascular disease. Cancer. 2000;88(9):2116-2121.

10. Meacham LR, Sklar CA, Li SW, et al. Diabetes mellitus in long-term survivors of childhood cancer increased risk associated with radiation therapy: a report for the childhood cancer survivor study. Arch Intern Med. 2009;169(15):1381-1388. doi:10.1001/archinternmed.2009.209

11. Holmqvist AS, Olsen JH, Andersen KK, et al. Adult life after childhood cancer in scandinavia: diabetes mellitus following treatment for cancer in childhood. Eur $J$ Cancer. 2014;50(6):1169-1175. doi:10.1016/j. ejca.2014.01.014

12. Mulrooney DA, Armstrong GT, Huang S, et al. Cardiac outcomes in adult survivors of childhood cancer exposed to cardiotoxic therapy. Ann Intern Med. 2016;164(2):93-101. doi:10.7326/M15-0424

13. Garmey EG, Liu Q, Sklar CA, et al. Longitudinal changes in obesity and body mass index among adult survivors of childhood acute lymphoblastic leukemia: a report from the Childhood Cancer Survivor Study. J Clin Oncol. 2008;26(28):4639-4645. doi:10.1200/JCO.2008.16.3527

14. Florin TA, Fryer GE, Miyoshi T, et al. Physical inactivity in adult survivors of childhood acute lymphoblastic leukemia: a report from the childhood cancer survivor study. Cancer Epidemiol Biomarkers Prev. 2007;16(7):1356-1363. doi:10.1158/1055-9965. EPI-07-0048

15. Schwartz CL. Long-term survivors of childhood cancer: the late effects of therapy. Oncologist. 1999;4(1):45-54.

16. NCD Risk Factor Collaboration. Worldwide trends in diabetes since 1980: a pooled analysis of 751 population-based studies with 4.4 million participants. Lancet. 2016;387(10027):1513-1530. doi:10.1016/S01406736(16)00618-8

17. Wang KW, Fleming A, Johnston DL, et al. Overweight, obesity and adiposity in survivors of childhood brain tumours: a systematic review and meta-analysis. Clin Obes. 2018;8(1):55-67. doi:10.1111/cob.12224 
18. Seuring T, Archangelidi O, Suhrcke M. The economic costs of type 2 diabetes: a global systematic review. Pharmacoeconomics. 2015;33 (8):811-831. doi:10.1007/s40273-015-0268-9

19. Moher D, Shamseer L, Clarke M, et al. Preferred reporting items for systematic review and meta-analysis protocols (PRISMA-P) 2015 statement. Syst Rev. 2015;4:1. doi:10.1186/2046-4053-4-1

20. Moher D, Stewart L, Shekelle P. Implementing PRISMA-P: recommendations for prospective authors. Syst Rev. 2016;5:15. doi:10.1186/ s13643-016-0289-2

21. Steliarova-Foucher E, Stiller C, Lacour B, Kaatsch P. International classification of childhood cancer. Cancer. 2005;103(7):1457-1467. doi:10.1002/cncr.20910

22. Urruticoechea A, Alemany R, Balart J, Villanueva A, Viñals F, Capellá G. Recent advances in cancer therapy: an overview. Curr Pharm Des. 2010;16(1):3-10. Review.

23. Robison LL, Armstrong GT, Boice JD, et al. The childhood cancer survivor study: a national cancer institute-supported resource for outcome and intervention research. J Clin Oncol. 2009;27 (14):2308. doi:10.1200/JCO.2009.22.3339

24. Liberati A, Altman DG, Tetzlaff J, et al. The PRISMA statement for reporting systematic reviews and meta-analyses of studies that evaluate healthcare interventions: explanation and elaboration. BMJ. 2009;339:b2700. doi:10.1136/bmj.b2651
25. Wang KW, Singh SK, Banfield L, de Souza RJ, ThabaneL, Samaan MC. Evaluating overweight and obesity prevalence in survivors of childhood brain tumors: a systematic review protocol. Syst. 2017;6(1):43.

26. EndNote [computer program]. Version X8.2: Clarivate Analytics; 2018

27. Hoy D, Brooks P, Woolf A, et al. Assessing risk of bias in prevalence studies: modification of anexisting tool and evidence of interrater agreement. J Clin Epidemiol. 2012;65(9):934-939. doi: 10.1016/j.jclinepi.2011.11.014.

28. Guyatt GH, Oxman AD, Kunz R, et al. GRADE guidelines: 7. Rating the quality of evidence-inconsistency. J Clin Epidemiol. 2011;64(12):1294 1302.

29. Egger M, Davey Smith G, Schneider M, Minder C. Bias in meta-analysis detected by a simple, graphical test. BMJ. 199713;315(7109):629.

30. Review Manager (RevMan) Version 5.3 [computer program]. Version 5.3. Copenhagen: The Cochrane Collaboration; 2014.

31. Neville KA, Cohn RJ, Steinbeck KS, Johnston K, Walker JL. Hyperinsulinemia, impaired glucose tolerance, and diabetes mellitus in survivors of childhood cancer: prevalence and risk factors. $J$ Clin Endocrinol Metab. 2006;91(11):4401-4407. doi:10.1210/jc.2006-0128

32. Talvensaari KK, Lanning M, Tapanainen P, Knip M. Long-term survivors of childhood cancer have an increased risk of manifesting the metabolic syndrome. J Clin Endocrinol Metab. 1996;81 (8):3051-3055. doi:10.1210/jcem.81.8.8768873
Adolescent Health, Medicine and Therapeutics

\section{Publish your work in this journal}

Adolescent Health, Medicine and Therapeutics is an international, peer-reviewed, open access journal focusing on health, pathology, and treatment issues specific to the adolescent age group. All aspects of health maintenance, preventative measures and disease treatment interventions are addressed within the journal and practitioners from all disciplines are invited to submit their work as well as healthcare researchers and patient support groups. The manuscript management system is completely online and includes a very quick and fair peerreview system. Visit http://www.dovepress.com/testimonials.php to read real quotes from published authors. 